\title{
Insomnia, Anxiety, and Depression in Patients First Diagnosed With Female Cancer
}

\author{
Dham Ho${ }^{1}$, Sun-Young $\mathrm{Kim}^{2}$, Soo In Kim¹, Sung-Youn $\mathrm{Kim}^{3 凶}{ }^{凶}$, and Weon-Jeong Lim ${ }^{2} \bowtie$ \\ ${ }^{1}$ Department of Psychiatry, Ewha Womans University Mokdong Hospital, Ewha Womans University College of Medicine, Seoul, Republic of Korea \\ ${ }^{2}$ Department of Psychiatry, Ewha Womans University Seoul Hospital, Ewha Womans University College of Medicine, Seoul, Republic of Korea \\ ${ }^{3} \mathrm{~S}$ Neuropsychiatric Clinic, Seoul, Republic of Korea
}

\begin{abstract}
Objective This study evaluated whether insomnia, anxiety, and depression differ by type of gynecological or breast cancer.
Methods From September 7, 2011, to July 14, 2015, this study included 232 patients who were diagnosed with gynecological or breast cancer for the first time. The severity of insomnia, anxiety, and depression was measured with the National Cancer Center Psychological Symptom inventory (NCC-PSI), a self-reported scale, at the first outpatient visit after surgery. Multivariate logistic regression analyses were used to identify which diagnosis was associated with significant symptom levels.

Results Patients with ovarian cancer and breast cancer reported more severe insomnia and problems with daily life compared with cervical cancer patients. Anxiety symptoms were more distressing among breast cancer patients than cervical cancer patients, and the degree of interference in daily life was severe. Finally, compared to those with cervical cancer, ovarian cancer and breast cancer patients reported more severe depression, and their daily life was disrupted more often than reported by cervical cancer patients.

Conclusion Many female cancer patients are suffering distress but are not looking for specialized care. Psychiatric approach in the early stages of cancer diagnosis is needed and will require overcoming the stigmas of mental illness and cancer.
\end{abstract}

Psychiatry Investig 2021;18(8):755-762

Key Words Cancer; Insomnia; Anxiety; Depression; National cancer center psychological symptom inventory.

\section{INTRODUCTION}

According to cancer statistics in Korea, the incidence rate of female cancer has been continuously increasing since 1999 by $6.6 \%, 5.3 \%$, and $1.9 \%$ in breast cancer, uterine cancer, and ovarian cancer, respectively. ${ }^{1}$ However, at the same time, with the developments in modern medicine, the survival rate of these female cancer patients has continued to increase; the relative survival rate of breast cancer, uterine cancer, ovarian cancer, and cervical cancer has risen to $14.0 \%, 5.3 \%, 4.8 \%$, and

Received: March 8, 2021 Revised: June 6, 2021

Accepted: June 24, 2021

$\triangle$ Correspondence: Weon-Jeong Lim, MD, PhD

Department of Psychiatry, Ewha Womans University Seoul Hospital, Ewha Womans University College of Medicine, 260 Gonghang-daero, Gangseo-gu, Seoul 07804, Republic of Korea

Tel: +82-2-6986-1676, Fax: +82-2-6986-3066, E-mail: psyweon@ewha.ac.kr

$\triangle$ Correspondence: Sung-Youn Kim, MD

S Neuropsychiatric Clinic, 399 Siheung-daero, Geumcheon-gu, Seoul 08584, Republic of Korea

Tel: +82-2-809-3088, Fax: +82-2-809-3097, E-mail: lesolle@naver.com

(a) This is an Open Access article distributed under the terms of the Creative Commons Attribution Non-Commercial License (https://creativecommons.org/licenses/by$\mathrm{nc} / 4.0$ ) which permits unrestricted non-commercial use, distribution, and reproduction in any medium, provided the original work is properly cited.
$1.9 \%$, respectively, with annual percentage change over the past five years (2013-2017) compared to 1993-1995. ${ }^{1}$ Therefore, it has become important to help and manage female cancer survivors to adapt well while living with cancer. In particular, there is a growing awareness that the mental health of cancer patients is important enough to affect not only quality of life, but also compliance with cancer treatment and, ultimately, the survival rate. The need for psychiatric evaluation and management of these patients is increasingly emphasized. ${ }^{2,3}$

The psychological difficulties of cancer patients during the diagnosis and treatment processes are defined by the National Comprehensive Cancer Network as 'Distress.'. 'Distress' is referred to as multidimensional and unpleasant emotional experiences that interfere with a patient's ability to effectively cope with cancer treatment. ${ }^{4}$ In Korea, the National Cancer Center (NCC) developed and provided a stress management recommendation for improving the quality of life of cancer patients in 2009, and used a Distress Thermometer (DT) to assess degree of distress. ${ }^{5,6}$ The National Cancer Center Psychological Symptom Inventory (NCC-PSI) evaluates specific distress items and life-interfering conditions and recommends 
emotional support or pharmacotherapy to medical staff depending on patient symptoms and severity. 5

Previous studies have shown that about $30 \%$ of breast and gynecological cancer patients complain of distress, and that there is a high risk of developing anxiety disorders or depression in the first year of cancer diagnosis. ${ }^{4,7,8}$ This indicates that many cancer patients are experiencing psychological distress, such as insomnia, anxiety, and depression, during the course of treatment. ${ }^{4,7,8}$

The reason cancer patients suffer from severe psychological distress is that, although the improvement of cancer treatment has increased recovery rate, having cancer implies the possibility of death and pain, which can create severe psychological distress. ${ }^{7,9}$ Furthermore, in cases of female cancer patients, for example, cervical cancer patients tend to be recognized as having many episodes of high-risk sex, early sexual experiences, and sexually transmitted infections related to risk factors. ${ }^{10,11}$ In addition, since breast cancer or other gynecological cancers can be associated with sexual dysfunction, this can lead to a "double stigma" of life-threatening diseases that affect sexual organs and sexual function. ${ }^{10,11}$

However, in practice, less than $10 \%$ of clinicians refer patients to psychiatric treatment, and more than half (55\%) of patients do not seek specialized care such as mental health services. ${ }^{7,12,13}$ Clover et al. ${ }^{14}$ reported that patients used to think that they could overcome mental problems on their own, or believed a cultural bias that suggested "psychiatric treatment is for strange people." These became obstacles to seeking psychiatric treatment, and the negative thoughts and beliefs of these patients could make it difficult for doctors to refer them to psychiatric treatment. ${ }^{\text {? }}$

Although several studies of female cancer patients have been conducted in Korea to understand their psychological difficulties, these studies have focused on the distress associated with side effects and stress in patients receiving chemotherapy or undergoing follow-up treatment after diagnosis with a female cancer, and there is a lack of research that examines the initial stress that patients feel after a female cancer diagnosis.

This study investigated the early distress that may occur in patients who are diagnosed with cervical cancer, endometrial cancer, ovarian cancer, and breast cancer from their first visit to an outpatient after surgery, but before chemotherapy or radiation therapy. The purpose of this study was to evaluate the severity of insomnia, anxiety, and depression according to sociodemographic characteristics and clinical variables that affect distress, the degree to which this stress interferes with daily life, and whether cancer patients seek specialized psychiatric care by medical staff due to the aforementioned symptoms.

\section{METHODS}

\section{Participants and procedures}

From September 7, 2011 to July 14, 2015, this study was conducted on patients aged 17 to 90 who were first diagnosed with gynecological or breast cancer at the Cancer Center of Ewha Womans University Mokdong Hospital. Subject distress was evaluated through the NCC-PSI questionnaire, a self-reported scale, at the first outpatient visit after surgery. Participants with poor judgment and understanding were excluded from the study to the extent that there was a change in their level of consciousness or if they had difficulty in completing the self-reported questionnaires. A total of 232 patients were finally analyzed, and the researchers explained the purpose and procedure of the study to the subjects and received written informed consent. This study received approval from the institution's Institutional Review Board (ECT 12-31A-46).

\section{Measurement tools}

\section{NCC-PSI}

The NCC-PSI is a self-reported measure of insomnia, anxiety, and depression commonly used in cancer patients in consideration of the domestic anti-cancer treatment environment, and patients are evaluated in the order of symptoms with relatively low stigma effects. ${ }^{6}$

The NCC-PSI consists of a Modified Distress Thermometer (MDT), which evaluates the severity of each symptom for insomnia, anxiety, and depressed mood, a Modified Impact Thermometer (MIT) that evaluates the effect of each symptom on daily life, and a question that indicates whether there is a symptom that the patient feels they need specialized care for from a medical professional. ${ }^{6}$

The MDT expresses the degree of insomnia, anxiety, and depression that a patient has felt over the past week using a total of 11 visual scales ranging from 0 points (no symptoms) to 10 points (symptoms of extreme severity). The MIT assesses if and how much each symptom interferes with daily life, and it is structured using the same visual scale from 0 points (no interference) to 10 points (complete interference). In Korea, Shim et al. ${ }^{6}$ evaluated the validity and reliability of the NCC-PSI, and in several studies, MDT and MIT each showed 4 points as the cut-off score that indicated clinically significant symptoms requiring an expert's help. Thus, it was suggested that there was significant distress if the score was 4 or more in one or more of the total 6 sub-items.

In addition, if specialized care for distress was needed, they were asked to select the item (insomnia, anxiety, or depressed mood) for which they needed specialized care. 


\section{Statistical analysis}

We grouped patients according to their type of cancer: cervical cancer, endometrial cancer, ovarian cancer, and breast cancer. For each patient group, the MDT and MIT scores for insomnia, anxiety, and depressed mood were compared between those with significantly elevated scores $(\geq 4)$ and those without significantly elevated scores $(<4)$. We used t-tests and chi-squared tests to compare these between score groups. Logistic regression analysis was used to examine the risk of significant levels of insomnia, anxiety, and depressed mood, and the degree of seeking specialized care by gynecological and breast cancer patients compared to those with cervical cancer. All regression models were adjusted for age, education, occupation, marital status, past medical history, hormone therapy status, and cancer family history. For statistical analysis, STATA version 14.0 (StataCorp LLC., College Station, TX, USA) was used, and the significance level ( $\mathrm{p}$-value) for determining statistical significance was set to 0.05 .

\section{RESULTS}

\section{Sociodemographic characteristics of patients with clinical level of insomnia, anxiety, and depressed mood}

The descriptive statistics for the study variables are presented in Table 1. In terms of age, the depressed group with MDTs of 4 or greater was younger than the not depressed group. For educational background, in all three types of distress (insomnia, anxiety, and depressed mood), the group who received an MDT or MIT score of 4 or greater showed a statistically significantly higher rate than the group who received a score less than 4 in the case of high school graduation or higher. Next, in terms of anxiety and depressive symptoms, the unmarried patient group had a higher rate of MDT or MIT scores of 4 or greater than did the group who received a score less than 4 . Additionally, people with a family history of cancer were more frequent in the group with an MDT or MIT scores of 4 or greater than in the group with a score less than 4 on depressed mood symptoms. There were no significant differences in the ratio of MDT and MIT scores of 4 or more or less with occupation, other medical history, or hormone therapy (Table 1).

\section{Comparison of insomnia report according to cancer type, degree of interference in daily life, and degree of need for specialized medical care}

With respect to MDT score on insomnia, ovarian cancer patients with MDT scores of 4 or greater were 6.11 times more likely to report significant symptom compared to cervical cancer patients $(\mathrm{OR}=6.11,95 \% \mathrm{CI}=2.06-18.15, \mathrm{p}=0.001)$. Breast cancer patients with MDT insomnia scores of 4 or greater were
7.99 times more likely to report significant symptom than those with cervical cancer $(\mathrm{OR}=7.99,95 \% \mathrm{CI}=2.10-30.45, \mathrm{p}=0.002)$. Ovarian cancer patients with MIT insomnia scores of 4 or greater were 4.43 times more likely to report symptom interference with daily life than those with cervical cancer $(\mathrm{OR}=$ $4.43,95 \% \mathrm{CI}=1.33-14.80, \mathrm{p}=0.015)$, and breast cancer patients were 6.11 times more likely to report symptom interference with daily life than cervical cancer patients $(\mathrm{OR}=6.11,95 \% \mathrm{CI}=$ 1.42-26.33, $\mathrm{p}=0.015)$. In terms of seeking specialized care for insomnia, no cancer patient group asked for specialized medical care more often than the cervical cancer patient group (Table 2).

\section{Comparison of anxiety report according to cancer type, degree of interference in daily life, and degree of need for specialized medical care}

With respect to MDT and MIT anxiety scores, breast cancer patients with scores of 4 or greater were 13.64 times more likely to report significant symptom $(\mathrm{OR}=13.64,95 \% \mathrm{CI}=$ 3.24-57.37, $\mathrm{p}<0.001)$ and 4.24 times more frequent to report interference with daily life $(\mathrm{OR}=4.24,95 \% \mathrm{CI}=1.14-15.87, \mathrm{p}=$ 0.032 ), respectively than patients with cervical cancer. In terms of seeking specialized care for anxiety symptoms, no cancer patient group showed significant results compared to the cervical cancer patient group (Table 3).

\section{Comparison of depressed mood report according to cancer type, degree of interference in daily life, and degree of need for specialized medical care}

With respect to MDT depressed mood score, ovarian cancer patients with scores of 4 or greater were 4.72 times more likely to report significant symptom than patients in the cervical cancer group ( $\mathrm{OR}=4.72,95 \% \mathrm{CI}=1.29-17.35, \mathrm{p}=0.019)$. Breast cancer patients with MDT scores of 4 or greater were 8.26 times more likely to report significant depressed symptom than cervical cancer patients $(\mathrm{OR}=8.26,95 \% \mathrm{CI}=1.89-$ $36.18, \mathrm{p}=0.005)$. For breast cancer patients with MIT depressed mood scores of 4 or greater were 4.78 times more likely to report symptom interference with daily life than cervical cancer patients $(\mathrm{OR}=4.78,95 \% \mathrm{CI}=1.12-20.47, \mathrm{p}=0.035)$. In terms of seeking specialized care for depressive symptoms, as with insomnia and anxiety symptoms, no cancer patient group showed significant results compared to the cervical cancer patient group (Table 4).

\section{DISCUSSION}

In this study, ovarian cancer patients reported more severe insomnia and depressive symptoms than cervical cancer patients, and insomnia particularly interfered with their daily 


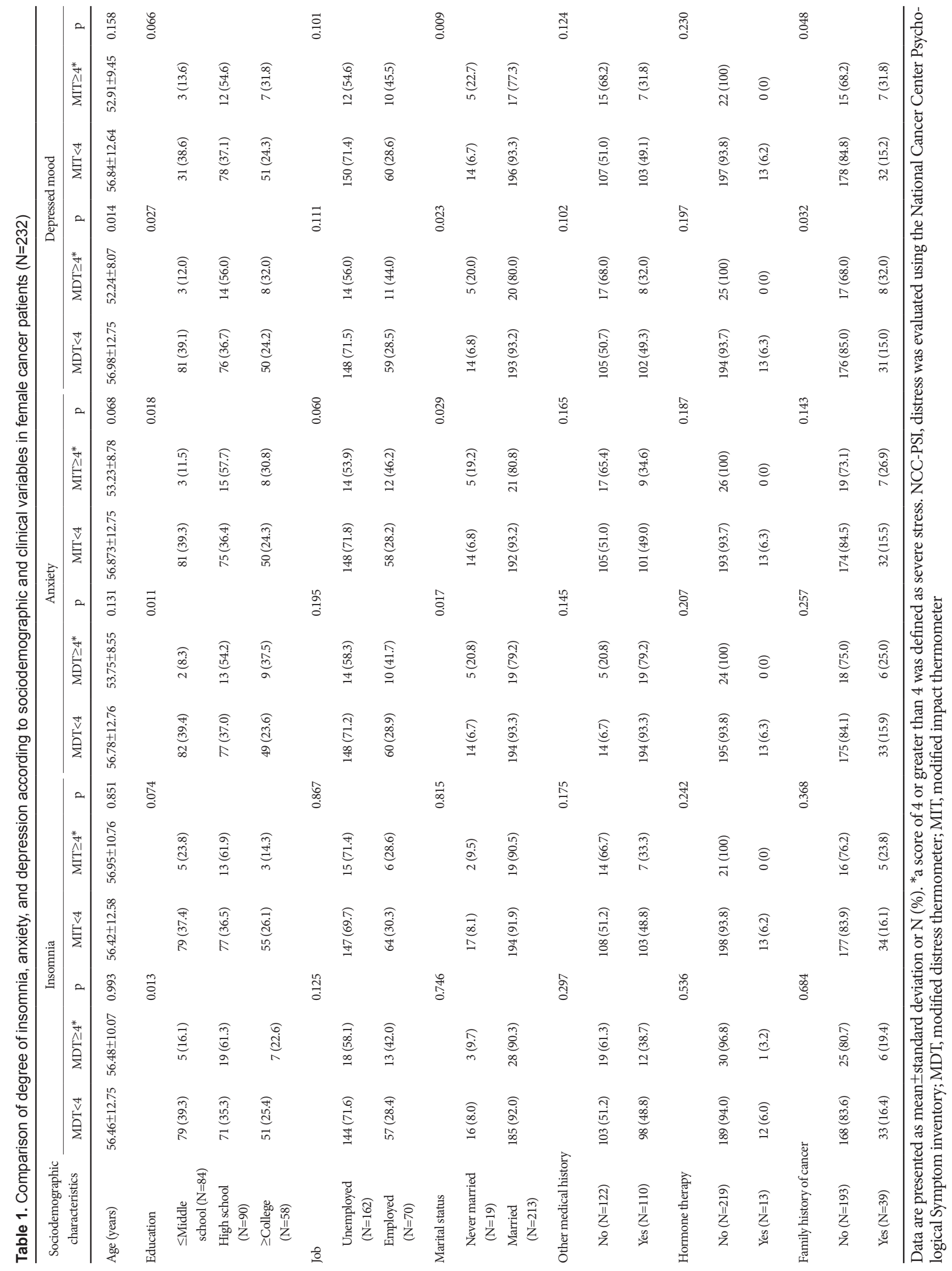


Table 2. Association of insomnia with clinical variables in multivariate logistic regression analyses

\begin{tabular}{|c|c|c|c|c|c|c|}
\hline \multirow{2}{*}{ Diagnosis } & \multicolumn{2}{|c|}{ MDT } & \multicolumn{2}{|l|}{ MIT } & \multicolumn{2}{|c|}{ Need for specialized care } \\
\hline & OR $(95 \% \mathrm{CI})^{*}$ & $\mathrm{p}$ & OR $(95 \% \mathrm{CI})^{*}$ & $\mathrm{p}$ & OR $(95 \% \mathrm{CI})^{*}$ & $\mathrm{p}$ \\
\hline Cervical cancer & 1 & & 1 & & 1 & \\
\hline Endometrial cancer & $1.22(0.31-4.77)$ & 0.779 & $0.82(0.15-4.54)$ & 0.819 & $0.38(0.41-3.54)$ & 0.395 \\
\hline Ovarian cancer & $6.11(2.06-18.15)$ & 0.001 & $4.43(1.33-14.80)$ & 0.015 & $0.90(0.16-5.01)$ & 0.900 \\
\hline Breast cancer & $7.99(2.10-30.45)$ & 0.002 & $6.11(1.42-26.33)$ & 0.015 & $4.04(0.74-22.04)$ & 0.107 \\
\hline
\end{tabular}

*adjustment for age, sex, education, job, marital status, past medical history, hormone therapy, and family history of cancer. OR, odds ratio; CI, confidence interval; MDT, modified distress thermometer; MIT, modified impact thermometer

Table 3. Association of anxiety with clinical variables in multivariate logistic regression analyses

\begin{tabular}{|c|c|c|c|c|c|c|}
\hline \multirow{2}{*}{ Diagnosis } & \multicolumn{2}{|c|}{ MDT } & \multicolumn{2}{|l|}{ MIT } & \multicolumn{2}{|c|}{ Need for specialized care } \\
\hline & OR $(95 \% \mathrm{CI})^{*}$ & $\mathrm{p}$ & OR $(95 \% \mathrm{CI})^{*}$ & $\mathrm{p}$ & OR $(95 \% \mathrm{CI})^{*}$ & $\mathrm{p}$ \\
\hline Cervical cancer & 1 & & 1 & & 1 & \\
\hline Endometrial cancer & $1.63(0.37-7.26)$ & 0.523 & $1.22(0.34-4.34)$ & 0.764 & $1.04(0.23-4.77)$ & 0.961 \\
\hline Ovarian cancer & $3.35(0.90-12.40)$ & 0.071 & $2.06(0.66-6.48)$ & 0.215 & $0.90(0.20-4.09)$ & 0.894 \\
\hline Breast cancer & $13.64(3.24-57.37)$ & 0.000 & $4.24(1.14-15.87)$ & 0.032 & $3.16(0.71-14.07)$ & 0.131 \\
\hline
\end{tabular}

*adjustment for age, sex, education, job, marital status, past medical history, hormone therapy, and family history of cancer. OR, odds ratio; CI, confidence interval; MDT, modified distress thermometer; MIT, modified impact thermometer

Table 4. Association of depressed mood with clinical variables in multivariate logistic regression analyses

\begin{tabular}{|c|c|c|c|c|c|c|}
\hline \multirow{2}{*}{ Diagnosis } & \multicolumn{2}{|c|}{ MDT } & \multicolumn{2}{|c|}{ MIT } & \multicolumn{2}{|c|}{ Need for specialized care } \\
\hline & OR $(95 \% \mathrm{CI})^{*}$ & $\mathrm{p}$ & OR $(95 \% \mathrm{CI})^{*}$ & $\mathrm{p}$ & OR $(95 \% \mathrm{CI})^{*}$ & $\mathrm{p}$ \\
\hline Cervical cancer & 1 & & 1 & & 1 & \\
\hline Endometrial cancer & $3.11(0.78-12.51)$ & 0.109 & $1.80(0.46-7.08)$ & 0.401 & $1.87(0.33-10.47)$ & 0.476 \\
\hline Ovarian cancer & $4.72(1.29-17.35)$ & 0.019 & $2.43(0.68-8.70)$ & 0.172 & $1.98(0.35-11.10)$ & 0.440 \\
\hline Breast cancer & $8.26(1.89-36.18)$ & 0.005 & $4.78(1.12-20.47)$ & 0.035 & $4.17(0.69-25.43)$ & 0.121 \\
\hline
\end{tabular}

*adjustment for age, sex, education, job, marital status, past medical history, hormone therapy, and family history of cancer. OR, odds ratio; CI, confidence interval; MDT, modified distress thermometer; MIT, modified impact thermometer

lives. Additionally, breast cancer patients suffered more subjective distress and disruptions to daily life due to insomnia, anxiety, and depressive symptoms compared with cervical cancer patients. However, none of the patients with breast cancer, ovarian cancer, or endometrial cancer sought medical care for these symptoms.

For sociodemographic characteristics of patients, first, the rate of complaining of significant distress was higher in the group of patients with a high school graduation or higher education. This result is inconsistent with previous studies showing that patients who are highly educated have a relatively lower prevalence of mental illness than do less educated patients, probably due to health promoting behaviors, such as exercise and healthy diet, and their tendency to have better awareness and knowledge related with health. ${ }^{15-19}$ The reason for this difference is that, for example, in Assari's ${ }^{19}$ study, 3,570 African American and 891 White men and women were studied, whereas in this study, only 232 women from East Asian were studied. The difference appears to be due to differences in gen- der, sample size, and ethnicity. In terms of marital status, those who complained of depression and anxiety were more often unmarried than were those who did not. This is consistent with the results of a prior study reporting that married patients showed psychological stability and a balanced life due to the support of spouses and family members who share the disease process compared to unmarried patients..$^{20,21}$

Although ovarian cancer is relatively rare compared to other types of cancer, it accounts for $4.9 \%$ of cancer-related deaths among American women, the fifth highest mortality rate among all types of cancer. ${ }^{10}$ Ovarian cancer does not have an effective method for early diagnosis, and it is difficult to detect early symptoms, so $59 \%$ of ovarian cancer patients are diagnosed with remote metastasis, and most of these experience recurrence within 18 months. ${ }^{22-24}$ According to the National Cancer Institute, the 5-year survival rate for ovarian cancer is $47.4 \%$, which is associated with severe symptom burden and poor quality of life as well as a vigorous treatment process. ${ }^{23,25,26} \mathrm{Be}-$ cause medical conditions such as pleural effusion, small bowel 
obstruction, and ascites, can occur in advanced and recurrent ovarian cancer patients as well as cancer-related fatigue and pain, which can lead to poor quality of life, ovarian cancer patients tend to lose a sense of control over their illness. ${ }^{10,25}$ In this situation, it seems that the negative prediction for treatment outcomes and a feeling of hopelessness can be attributed to this patient group's poor prognosis. As the treatment progresses, distress may increase due to the side effects of the treatment itself and accumulation of fatigue, so it seems necessary to pay more attention to ovarian cancer patients, among other gynecological cancer patients.

Breast cancer accounts for 33\% of all female cancers and $20 \%$ of female cancer-related deaths. ${ }^{27}$ When breast cancer is diagnosed, the initial reaction is related to the fear of pain and death, similar to other cancers, and the subsequent reactions are more representative of motherhood, sexuality, and aesthetics than the surgical treatment itself which will cause breast loss. ${ }^{27,28}$ Therefore, secondary breast loss can cause loss of attractiveness, fertility, libido, and overall femininity. ${ }^{27}$ In addition, breast cancer patients may experience a decrease in their quality of life due to chemotherapy, radiation therapy, and additional surgical treatment, and in this treatment process, many patients experience various complications as they suffer from amenorrhea or early menopause. ${ }^{27,29}$ Hormone therapy is sometimes recommended to prevent osteoporosis or other complications in cases of early menopause or long-term amenorrhea due to anticancer drugs, but hormone therapy is contraindicated in breast cancer patients, so it is often difficult to provide adequate treatment. ${ }^{27,29}$ Also, studies show that the risk of depression can increase when estrogen decreases, and that the more severe are menopause symptoms such as hot flushes or nighttime sweats, the higher is the risk of depression and anxiety. ${ }^{30,31}$ In addition to amenorrhea and early menopause, breast cancer patients may face various gynecological problems such as vaginal dryness and endometrial abnormalities while using Tamoxifen or an aromatase inhibitor after surgery. ${ }^{10,30,32-34}$ Tamoxifen has both estrogen agonists and antagonists, with estrogen receptors present in the brain associated with cognition and emotion, and changes in neurotransmitters such as serotonin, dopamine, and acetylcholine can occur through estrogen receptors. ${ }^{31,35}$ Therefore, most breast cancer patients exhibit psychological reactions such as rejection, anger, and extreme fear of their disease characteristics and treatment process. Further studies are necessary to determine if breast cancer patients experience greater emotional distress than do other gynecological cancer patients.

Despite the fact that many cancer patients suffer from distress, this study found that no cancer patients are significantly seeking specialized care for treatment of their psychiatric symptoms. There are several possibilities for this outcome.
First, mental illness has been subject to fear and threat for centuries, and with the perception that it is potentially due to personal negligence, this negative perception can reduce the credibility of beneficial psychiatric treatment and raise concerns that psychiatric treatment can have negative social consequences for individuals. ${ }^{11,36} \mathrm{Next}$, stigma about cancer itself can also hamper patients in their search for psychiatric treatment. Holland et al. ${ }^{11}$ are aware of the uncertainty and incurable diseases of cancer that can lead to punishment, retribution, or fate for the past and present, which can have a significant impact on the course of cancer treatment. In fact, not only patients but also doctors consider the psychiatric intervention they experience as inappropriate "medicalization" due to dismissing the patient's stress as a normal response to cancer or an expected result. ${ }^{11,37}$ Factors with low interest in psychiatric intervention include severe distress symptoms and low social support, low levels of disease knowledge, and avoidance tendencies at the individual level, however, they would benefit the most from mental intervention. ${ }^{11,37,38}$

This study analyzed female cancer patients for the first time and found that insomnia, anxiety, and depression symptoms appeared in a fairly large proportion. This suggests that, in female cancer patients, not only physical problems, but also mental problems should be evaluated and managed at an early stage of diagnosis. Various factors such as psychosocial stress or somatic symptoms that adversely can affect the mental health of cancer patients should be accurately evaluated. In particular, since somatic symptoms can occur or worsen due to mental problems, psychiatric symptoms need to be evaluated and managed from a psychosomatic point of view. The results of this study showed that the rate of seeking medical help for psychiatric symptoms was low despite the poor mental health of female cancer patients. Therefore, social efforts to reduce stigma on psychiatry and cancer are needed, and it will be helpful to encourage patients to actively receive specialized mental health care even in the early stages of cancer.

The limitations of this study are as follows. First, since this study only analyzed breast cancer, cervical cancer, endometrial cancer, and ovarian cancer patients, it is difficult to generalize our results to all female cancer patients. Further studies on a larger number of female cancer patients need to be conducted to comprehensively evaluate the distress of female cancer patients. Second, it was not possible to rule out patients with pre-existing mental disorders. Third, in this study, only a self-reported evaluation scale was used to evaluate psychological symptoms, and objective and structured interviews were not conducted, so it is difficult to be sure of the objectivity and validity of patient psychiatric conditions in this study.

However, despite these limitations, this study measured distress at the first outpatient visit after surgery for patients with 
female cancers diagnosed for the first time. We tried to analyze the initial distress that can be the result of the cancer diagnosis itself, excluding the effects of various clinical variables such as chemotherapy, radiation therapy, and anti-hormone therapy. This study investigated the distress that may occur in the early stages of the treatment process of female cancer patients, and specifically evaluated the most common symptoms of distress, i.e., insomnia, anxiety, and depressed mood. This is significant in that we sought a multidisciplinary and integrated approach from the beginning of treatment for female cancer patients by analyzing the association of sociodemographic characteristics and clinical variables that influence each kind of distress.

Based on the results of this study, a prospective study that attempts therapeutic interventions for distress that may occur in the early stages of treatment in cancer patients is needed. Furthermore, it is necessary to investigate how early psychiatric interventions affect cancer patient overall treatment course and prognosis. It will also be necessary to analyze stress-impacting factors that occur from the first-time cancer diagnosis through follow-up surveys throughout the treatment process, to establish standardized treatment guidelines for each period of female cancer treatment to increase patient compliance and prognosis, and improve patient quality of life.

\section{Availability of Data and Material}

The datasets generated or analyzed during the study are available from the corresponding author on reasonable request.

\section{Conflicts of Interest}

The authors have no potential conflicts of interest to disclose.

\section{Author Contributions}

Conceptualization: Weon-Jeong Lim, Sung-Youn Kim. Data curation: Weon-Jeong Lim. Investigation: Dham Ho, Sun-Young Kim. Methodology: Dham Ho, Sun-Young Kim. Project administration: Soo In Kim, Weon-Jeong Lim. Supervision: Sun-Young Kim. Writing_original draft: Dham Ho. Writing_review \& editing: all authors.

\section{ORCID iDs}

Dham Ho

Sun-Young Kim

Soo In Kim

Sung-Youn Kim

https://orcid.org/0000-0001-8963-754X

https://orcid.org/0000-0003-3705-9746

https://orcid.org/0000-0001-6909-5034

https://orcid.org/0000-0003-3214-4263

Weon-Jeong Lim https://orcid.org/0000-0002-2100-4233

\section{Funding Statement}

The research did not receive any specific grant from funding agencies in the public, commercial, or not-for-profit sectors.

\section{REFERENCES}

1. Hong S, Won YJ, Park YR, Jung KW, Kong HJ, Lee ES. Cancer statistics in Korea: incidence, mortality, survival, and prevalence in 2017. Cancer Res Treat 2020;52:335-350.

2. Boyes AW, Girgis A, D’Este CA, Zucca AC, Lecathelinais C, Carey ML.
Prevalence and predictors of the short-term trajectory of anxiety and depression in the first year after a cancer diagnosis: a population-based longitudinal study. J Clin Oncol 2013;31:2724-2729.

3. Han S, Shin I, Kim Y. Factors that influence quality of life in cancer patients. Korean J Health Promot Dis Prev 2009;9:33-40.

4. Holland JC, Andersen B, Breitbart WS, Buchmann LO, Compas B, Deshields TL, et al. Distress management. J Nat Compr Cancer Netw 2013;11:190-209.

5. Kim J, Kang J, Kim J, Kim T, Kim H, Kim H. Development of Recommendations for Distress Management toward Improvement of Quality of Life in Cancer Patients. Seoul: Ministry of Health \& Welfare; 2009.

6. Shim EJ, Hahm BJ, Yu ES, Kim HK, Cho SJ, Chang SM, et al. Development and validation of the National Cancer Center Psychological Symptom Inventory. Psychooncology 2017;26:1036-1043.

7. Anuk D, Özkan M, Kizir A, Özkan S. The characteristics and risk factors for common psychiatric disorders in patients with cancer seeking help for mental health. BMC Psychiatry 2019;19:269.

8. Bjerkeset E, Röhrl K, Schou-Bredal I. Symptom cluster of pain, fatigue, and psychological distress in breast cancer survivors: prevalence and characteristics. Breast Cancer Res Treat 2020;180:63-71.

9. Boyle P, Levin B. World Cancer Report 2008. IARC Press, International Agency for Research on Cancer; 2008.

10. Korean Society of Gynecologic Oncology, Gynecologic Oncology. Seoul: KOONJA; 2020.

11. Holland JC, Kelly BJ, Weinberger MI. Why psychosocial care is difficult to integrate into routine cancer care: stigma is the elephant in the room. J Nat Compr Cancer Netw 2010;8:362-366.

12. Holland JC. IPOS Sutherland Memorial Lecture: an international perspective on the development of psychosocial oncology: overcoming cultural and attitudinal barriers to improve psychosocial care. Psychonology 2004;13:445-459.

13. Forsythe LP, Kent EE, Weaver KE, Buchanan N, Hawkins NA, Rodriguez JL, et al. Receipt of psychosocial care among cancer survivors in the United States. J Clin Oncol 2013;31:1961-1969.

14. Clover KA, Mitchell AJ, Britton B, Carter G. Why do oncology outpatients who report emotional distress decline help? Psychooncology 2015; 24:812-818.

15. Ennis NE, Hobfoll SE, Schröder KE. Money doesn't talk, it swears: How economic stress and resistance resources impact inner-city women's depressive mood. Am J Community Psychol 2000;28:149-173.

16. Wang J, Geng L. Effects of socioeconomic status on physical and psychological health: lifestyle as a mediator. Int J Environ Res Public Health 2019;16:281.

17. Mackenbach JP. Socioeconomic inequalities in health in the Netherlands: impact of a five year research programme. BMJ 1994;309:14871491.

18. Adler NE, Boyce T, Chesney MA, Cohen S, Folkman S, Kahn RL, et al. Socioeconomic status and health: the challenge of the gradient. Am Psychol 1994;49:15-24.

19. Assari S. Social determinants of depression: the intersections of race, gender, and socioeconomic status. Brain Sci 2017;7:156.

20. Lee YS. Psychosocial experience in post-mastectomy women. Korean J Soc Welfare 2007;59:99-124.

21. Yucel D. Work-family balance and marital satisfaction: the mediating effects of mental and physical health. Soc Ment Health 2017;7:175-195.

22. Siegel RL, Miller KD, Jemal A. Cancer statistics, 2019. CA Cancer J Clin 2019;69:7-34.

23. Hill EM, Hamm A. Intolerance of uncertainty, social support, and loneliness in relation to anxiety and depressive symptoms among women diagnosed with ovarian cancer. Psychooncology 2019;28:553-560.

24. Jayson GC, Kohn EC, Kitchener HC, Ledermann JA. Ovarian cancer. Lancet 2014;384:1376-1388.

25. Roland KB, Rodriguez JL, Patterson JR, Trivers KF. A literature review of the social and psychological needs of ovarian cancer survivors. Psychooncology 2013;22:2408-2418. 
26. Trivers KF, Patterson JR, Roland KB, Rodriguez JL. Issues of ovarian cancer survivors in the USA: a literature review. Support Care Cancer 2013;21:2889-2898.

27. İzci F, İlgün AS, Fındıklı E, Özmen V. Psychiatric symptoms and psychosocial problems in patients with breast cancer. J Breast Health 2016; 12:94-101.

28. Baider L, Andritsch E, Uziely B, Ever-Hadani P, Goldzweig G, Hofmann $\mathrm{G}$, et al. Do different cultural settings affect the psychological distress of women with breast cancer? A randomized study. Eur J Cancer Care (Engl) 2003;12:263-273.

29. Bardwell WA, Profant J, Casden DR, Dimsdale JE, Ancoli-Israel S, Natarajan L, et al. The relative importance of specific risk factors for insomnia in women treated for early-stage breast cancer. Psychooncology 2008;17:9-18.

30. Moon Z, Hunter MS, Moss-Morris R, Hughes LD. Factors related to the experience of menopausal symptoms in women prescribed tamoxifen. J Psychosom Obstet Gynaecol 2017;38:226-235.

31. Novick AM, Scott AT, Epperson CN, Schneck CD. Neuropsychiatric Effects of Tamoxifen: Challenges and Opportunities. Front Neuroendocrinol 2020:59;100869.

32. Gupta P, Sturdee D, Palin S, Majumder K, Fear R, Marshall T, et al. Menopausal symptoms in women treated for breast cancer: the prevalence and severity of symptoms and their perceived effects on quality of life. Climacteric 2006;9:49-58.

33. Garreau JR, Delamelena T, Walts D, Karamlou K, Johnson N. Side effects of aromatase inhibitors versus tamoxifen: the patients' perspective. Am J Surg 2006;192:496-498.

34. Boehm DU, Lebrecht A, Eckhardt T, Albrich S, Schmidt M, Siggelkow W, et al. Quality of life and adjuvant tamoxifen treatment in breast cancer patients. Eur J Cancer Care (Engl) 2009;18:500-506.

35. Newhouse P, Albert K, Astur R, Johnson J, Naylor M, Dumas J. Tamoxifen improves cholinergically modulated cognitive performance in postmenopausal women. Neuropsychopharmacology 2013;38:2632-2643.

36. Zhang Z, Sun K, Jatchavala C, Koh J, Chia Y, Bose J, et al. Overview of stigma against psychiatric illnesses and advancements of anti-stigma activities in six Asian societies. Int J Environ Res Public Health 2020; $17: 280$.

37. Stanton AL. How and for whom? Asking questions about the utility of psychosocial interventions for individuals diagnosed with cancer. J Clin Oncol 2005;23:4818-4820.

38. Merckaert I, Libert Y, Messin S, Milani M, Slachmuylder JL, Razavi D. Cancer patients' desire for psychological support: prevalence and implications for screening patients' psychological needs. Psychooncology 2010;19:141-149. 